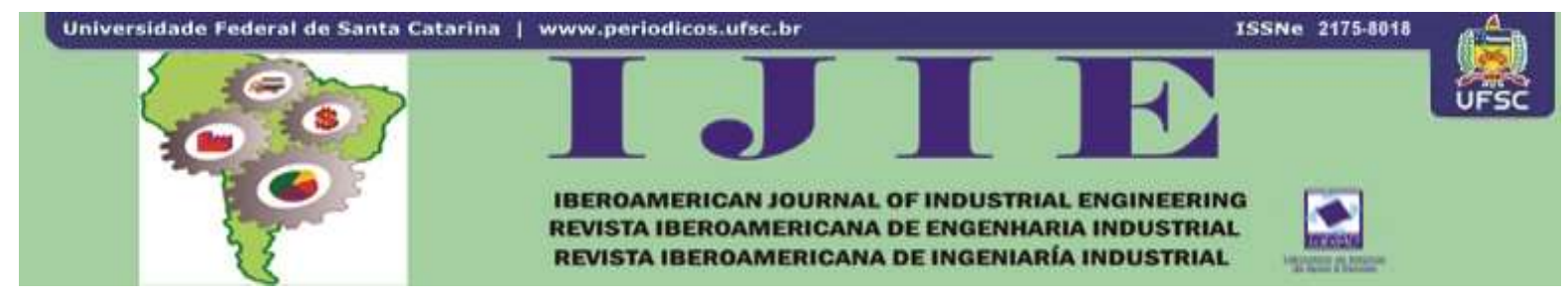

\title{
PRODUCCIÓN DE ETANOL A PARTIR DE BIOMASA LIGNOCELULÓSICA: ESTIMACIÓN DINÁMICA DE PARÁMETROS CON ENFOQUE SIMULTÁNEO
}

\author{
Federico Ezequiel Andersen ${ }^{1}$ \\ Marta Susana Moreno ${ }^{2}$ \\ Maria Soledad Díaz ${ }^{3}$
}

\begin{abstract}
RESUMEN: En este trabajo se lleva a cabo la estimación de parámetros en modelos cinéticos correspondientes a las diferentes operaciones involucradas en la producción bioquímica de etanol lignocelulósico. El modelo resultante se representa con un sistema de ecuaciones algebraico-diferenciales (DAE), que incluye las principales variables del proceso. El problema de estimación de parámetros se resuelve mediante un enfoque simultáneo, en el que el DAE se convierte en un sistema algebraico, mediante la técnica de colocación ortogonal sobre elementos finitos. Así, estimar los parámetros del modelo implica minimizar una función objetivo de cuadrados mínimos ponderados sujeta a restricciones algebraicas. Los modelos cinéticos se pueden emplear en futuros trabajos de diseño y simulación de plantas productoras de etanol. En cada modelo se lograron ajustes satisfactorios de los datos experimentales obtenidos de bibliografía.
\end{abstract}

Palabras clave: Estimación de parámetros. Optimización dinámica. Colocación ortogonal. Etanol lignocelulósico.

\section{INTRODUCCIÓN}

La biomasa lignocelulósica es la más abundante y económica de las materias primas en la naturaleza utilizadas para la producción del denominado bioetanol de segunda generación. La misma contiene una mezcla heterogénea de biopolímeros proveniente de la pared celular

\footnotetext{
${ }^{1} \mathrm{PhD}$ in Chemical Engineering, Planta Piloto de Ingeniería Química (PLAPIQUI), Consejo Nacional de Investigaciones Científicas y Técnicas (CONICET) - Universidad Nacional del Sur, Camino La Carrindanga km 7, 8000 Bahía Blanca, Argentina. E-mail: fandersen@plapiqui.edu.ar.

$2 \mathrm{PhD}$ in Chemical Engineering, Assistant Professor Universidad Nacional del Sur, Assistant Researcher Planta Piloto de Ingeniaria Quimica, Centro Científico Tecnológico (PLAPIQUI-CONICET), Camino La Carrindanga km 7 (8000) Bahía Blanca/Argentina. E-mail: smoreno@plapiqui.edu.ar.

3 Doctora en Ingeniería Química, Planta Piloto de Ingeniería Química (PLAPIQUI), Consejo Nacional de Investigaciones Científicas y Técnicas (CONICET) - Universidad Nacional del Sur, Camino La Carrindanga km 7, 8000 Bahía Blanca, Argentina. E-mail: sdiaz@plapiqui.edu.ar.
}

Iberoamerican Journal of Industrial Engineering, Florianópolis, SC, Brasil, v. 5, n. 10, p. 219231, 2013. 
vegetal: celulosa, hemicelulosa y lignina. Los dos primeros polímeros constituyen fuentes potenciales de producción de azúcares (principalmente glucosa y xilosa). Sin embargo, estos compuestos no son de fácil acceso, ya que se encuentran dentro de una estructura difícil de destruir, conformada principalmente por la lignina.

Para producir etanol a partir de materiales lignocelulósicos, por conversión bioquímica, primero las fracciones de celulosa y hemicelulosa se deben transformar en azúcares. Esto se logra mediante una etapa de pre-tratamiento que pretende remover la lignina e hidrolizar la hemicelulosa a azúcares. Esta operación hace más susceptible el ataque de la celulosa por parte de las enzimas para liberar glucosa en la hidrólisis enzimática. Finalmente, los azúcares se fermentan a etanol, mientras que la fracción de lignina se separa y se emplea como fuente de energía para el proceso.

Aunque se han propuesto diferentes tecnologías de pre-tratamiento, la hidrólisis ácida diluida es la preferida para aplicaciones industriales debido a su simplicidad y altos rendimientos. Sin embargo, este proceso tiene como desventaja la producción de compuestos tóxicos que pueden inhibir a los microorganismos empleados en la fermentación posterior. Por lo tanto, luego de este pre-tratamiento se requiere una etapa de detoxificación del hidrolizado, donde los compuestos tóxicos se convierten a productos menos inhibitorios. El remanente sólido de la hidrólisis ácida se trata con enzimas específicas que permiten extraer los azúcares de seis carbonos de la celulosa. El producto de esta hidrólisis enzimática junto a la corriente detoxificada se envían a una etapa de co-fermentación para la obtención de etanol.

En general, modelos cinéticos propuestos en bibliografía (RANATUNGA et al.; 1997; LEKSAWASDI; JOACHIMSTHAL; ROGERS, 2001; LAVARACK; GRIFFIN; RODMAN, 2002; PURWADI; NIKLASSON; TAHERZADEH, 2004) para las diferentes etapas que conforman el proceso de producción de etanol lignocelulósico, no incluyen un análisis de las variables de proceso necesarias para cuantificar la influencia de posibles cambios que puedan realizarse en el modelo y así poder optimizar el mismo.

En este trabajo se realiza la estimación de parámetros cinéticos en modelos que incluyen las principales variables de proceso, como la temperatura, el $\mathrm{pH}$ y la concentración, en las operaciones unitarias de hidrólisis ácida diluida, detoxificación y co-fermentación. Los modelos propuestos se ajustan empleando datos experimentales de bibliografía (PURWADI; NIKLASSON; TAHERZADEH, 2004; CASSALES et al., 2011; GUTIERREZ-PADILLA; KARIM, 2005). El problema dinámico de estimación de parámetros, formulado para cada una de las etapas mencionadas, se modela como un sistema DAE que luego se convierte a un 
problema de programación no lineal (NLP) de gran escala mediante la técnica de colocación ortogonal sobre elementos finitos. El modelo NLP resultante se resuelve con métodos de punto interior (IP) del solver IPOPT (WÄCHTER; BIEGLER, 2006) que utiliza técnicas de programación cuadrática sucesiva (SQP) dentro de la plataforma GAMS (BROOKE et al., 2012). Los modelos presentados a continuación se encuentran detallados en forma completa en Andersen, Moreno y Díaz (2011), Moreno, Andersen y Díaz (2013).

\section{METODOLOGÍA APLICADA}

En este trabajo, los procesos se representan mediante modelos dinámicos constituidos por sistemas de ecuaciones algebraico-diferenciales (DAE). De esta manera, el problema de estimación de parámetros se formula como un problema de optimización dinámico restringido, con una forma general como se muestra en $\mathbf{P 1}$.

Aquí, los índices $e, j$ y $m$ corresponden al experimento realizado, la variable diferencial y el punto experimental, respectivamente. El vector $\mathbf{z}$ corresponde al valor calculado de las variables diferenciales, en tanto que $\hat{\mathbf{z}}$ corresponde a los valores medidos de las variables diferenciales. El vector $\mathbf{p}$ representa los parámetros a estimar, el vector $\mathbf{y}$ representa las variables algebraicas y $\mathbf{z}^{\mathbf{0}}$ representa el vector de las condiciones iniciales para las variables diferenciales. La matriz diagonal $\mathbf{W}$ contiene los pesos que afectan a la función objetivo. La variable $t$ representa el tiempo, f representa el vector de términos independientes de las ecuaciones diferenciales y $\mathbf{g}$ es el vector de restricciones algebraicas. Se considera que los $N E$ experimentos son realizados bajo condiciones diferentes y que se miden $J_{e}$ variables diferenciales en cada uno de ellos, colectando un total de $M_{j e}$ puntos en cada experiencia para cada variable diferencial.

La función objetivo a minimizar es la suma de los cuadrados de los residuos, ponderados por la inversa de la varianza del error de medición. Se utiliza un enfoque de colocación simultáneo que discretiza totalmente los perfiles de las variables diferenciales respecto del tiempo, convirtiendo el sistema dinámico en un conjunto de ecuaciones algebraicas. Para esto se utiliza el método de colocación ortogonal sobre elementos finitos (BIEGLER; ZAVALA, 2009).

$$
\min \theta=\sum_{e=1}^{N E} \sum_{j=1}^{J_{e}} \sum_{m=1}^{M_{j e}}\left(\hat{z}_{e j m}-z_{e j m}\right)^{T} W^{-1}\left(\hat{z}_{e j m}-z_{e j m}\right)
$$

sujeto a: 


$$
\begin{gathered}
\frac{d \mathrm{z}}{d t}=\mathrm{f}(\mathrm{z}, \mathrm{y}, \mathrm{p}, t) \\
\mathrm{g}(\mathrm{z}, \mathrm{y}, \mathrm{p}, t)=0 \\
\mathrm{z}\left(t_{0}\right)=\mathrm{z}^{0} \quad t \in\left[t_{0}, t_{F}\right] \\
\mathrm{z}^{L} \leq \mathrm{z} \leq \mathrm{z}^{U} \\
\mathrm{p}^{L} \leq \mathrm{p} \leq \mathrm{p}^{U}
\end{gathered}
$$

\section{ETAPA DE HIDRÓLISIS ÁCIDA DILUIDA}

Este pre-tratamiento consiste en la hidrólisis de la fracción hemicelulósica empleando ácidos diluidos (concentraciones por debajo de $5 \%$ en peso) como catalizadores, a temperaturas moderadas $\left(110\right.$ a $\left.230^{\circ} \mathrm{C}\right)$.

Se han presentado distintos modelos cinéticos para describir las reacciones de hidrólisis. Este trabajo se basa en el esquema de reacción más simple propuesto por Lavarack et al. (2002). Estos autores asumen que la velocidad de reacción es de primer orden con respecto a los reactivos en cada una de las reacciones consideradas. Además, consideran que la constante de velocidad de reacción sigue una relación del tipo Arrhenius, modificada por una dependencia potencial con la concentración de ácido.

Las reacciones de hidrólisis consideradas en este trabajo se muestran en la Figura 1. Se asume que la formación de xilosa, arabinosa, glucosa y lignina soluble se corresponden con el esquema propuesto por Lavarack et al. (2002). Debido a las bajas concentraciones de ácido, se asume que la fracción celulósica permanece inalterada y que la glucosa producida se obtiene a partir del ácido glucurónico presente en las cadenas laterales del xilano. Se propone además que el furfural se obtiene a partir de la descomposición de xilano y arabiano.

Figura 1 - Reacciones químicas de la hidrólisis ácida

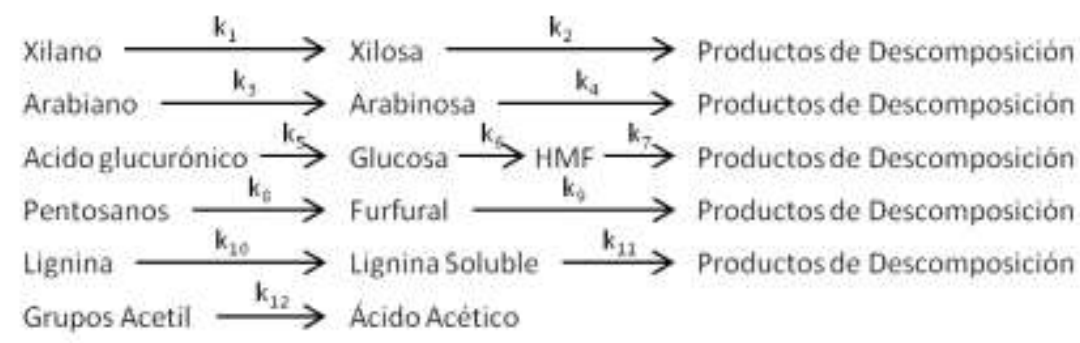

En este trabajo, se extiende el modelo anterior para incluir la producción de ácido acético debido a la liberación de los grupos acetil unidos a la cadena principal del xilano. 
Asimismo, se incluye la degradación de glucosa a hidroximetilfurfural (HMF) que a su vez, se descompone en un paso posterior. Basados en el mecanismo propuesto en la Figura 1, los principales balances de masa planteados son los que se muestran en las Ecuaciones 1-7:

$$
\begin{gathered}
\frac{d C_{X n}}{d t}=-k_{1} C_{X n}-k_{8} C_{X n} \\
\frac{d C_{X y}}{d t}=k_{1} C_{X n} \emptyset \rho-k_{2} C_{X y} \\
\frac{d C_{F}}{d t}=k_{8}\left(C_{X n}+C_{A n}\right) \emptyset \rho-k_{9} C_{F} \\
\frac{d C_{H M F}}{d t}=k_{6} C_{G}-k_{7} C_{H M F} \\
\frac{d C_{A c G}}{d t}=-k_{12} C_{A c G} \\
\frac{d C_{A c H}}{d t}=k_{12} C_{A c G} \\
k_{i}=k_{i}{ }^{0} C_{A c}^{n_{i}} \exp \left(-E_{i} / R T\right) \quad \forall i
\end{gathered}
$$

donde $\phi$ representa la relación entre material sólido y líquido en la operación de hidrólisis [kg sólido/kg líquido], $\rho$ es la densidad del hidrolizado [kg líquido/L], $\mathrm{C}_{\mathrm{i}}$ representa la concentración del componente $i$ expresada en $\mathrm{g} / \mathrm{kg}$ sólido para las especies presentes en la biomasa sólida y g/L para los productos solubilizados en el hidrolizado. La Ecuación 7 permite calcular la constante de velocidad de cada reacción con una ecuación tipo Arrhenius modificada.

Por lo tanto, el problema de estimación de parámetros consta de 12 Ecuaciones diferenciales y 12 ecuaciones algebraicas. El vector de parámetros p corresponde a los factores pre-exponenciales $k_{i}{ }^{0}$, los exponentes $n_{i}$ y las energías de activación $E_{i}$, para cada reacción $i$.

Por lo tanto, el problema de estimación de parámetros consta de 12 Ecuaciones diferenciales y 12 Ecuaciones algebraicas. El vector de parámetros p corresponde a los factores pre-exponenciales $k_{i}{ }^{0}$, los exponentes $n_{i}$ y las energías de activación $E_{i}$, para cada reacción $i$.

Tabla 1 - Valores de desviación media para compuestos que participan de la hidrólisis ácida

\begin{tabular}{lccccccc}
\hline & Xilosa & Arabinosa & Glucosa & Furfural & HMF & Ácido Acético & Lignina Soluble \\
\hline $\begin{array}{l}\text { Desviación } \\
\text { Media }\end{array}$ & $-2.6 \%$ & $4.5 \%$ & $6.1 \%$ & $-1.9 \%$ & - & $8.2 \%$ & $2.9 \%$ \\
\hline
\end{tabular}

Iberoamerican Journal of Industrial Engineering, Florianópolis, SC, Brasil, v. 5, n. 10, p. 219-231, 2013. 
Los datos experimentales necesarios para llevar a cabo esta estimación de parámetros han sido extraídos de Cassales et al. (2011). Estos autores realizaron 9 experiencias, a diferentes temperaturas y concentraciones de ácido sulfúrico, sobre $60 \mathrm{~min}$ de reacción tomando muestras cada $10 \mathrm{~min}$.

Finalmente, el sistema DAE consta de 108 ecuaciones diferenciales, 6 puntos experimentales para cada ecuación diferencial más la condición inicial y 15 parámetros a estimar. El modelo NLP resultante tiene 2.584 ecuaciones y 2.599 variables.

En la Tabla 2 se detallan los valores óptimos de los parámetros, mientras que en la Tabla 1 se muestran los valores de desviación media calculados con las concentraciones óptimas de algunos compuestos.

Tabla 2 - Valores óptimos de los parámetros más influyentes en el modelo de hidrólisis ácida

\begin{tabular}{cccccc}
\hline Parámetro & Valor óptimo & Parámetro & Valor óptimo & Parámetro & Valor óptimo \\
\hline$k_{1}{ }^{0}$ & $4,09110^{9}$ & $E_{3}$ & 35,092 & $n_{10}$ & 0,100 \\
$n_{1}$ & 1,391 & $k_{5}{ }^{0}$ & $1,86010^{6}$ & $E_{10}$ & 15,240 \\
$E_{1}$ & 76,361 & $n_{5}$ & 0,616 & $k_{12}{ }^{0}$ & $1,85710^{17}$ \\
$k_{3}{ }^{0}$ & $8,45410^{4}$ & $E_{5}$ & 45,670 & $n_{12}$ & 0,095 \\
$n_{3}$ & 0,984 & $k_{10}{ }^{0}$ & $1,02510^{3}$ & $E_{12}$ & 144,055 \\
\hline
\end{tabular}

\section{ETAPA DE DETOXIFICACIÓN: TRATAMIENTO CON CA(OH $)_{2}$}

La corriente líquida (hidrolizado) proveniente de la hidrólisis ácida diluida, se envía a una etapa de detoxificación para extraer compuestos tóxicos, tales como furfural y HMF, inhibidores de la etapa de fermentación. La producción de estos compuestos en la hidrólisis ácida se encuentra fuertemente influenciada por condiciones de temperatura, tiempo de reacción y concentración de ácido.

Existen numerosas técnicas para eliminar estos compuestos (tratamiento con $\mathrm{Ca}(\mathrm{OH})_{2}$, intercambio aniónico, evaporación, tratamiento con enzimas y/o bacterias, entre otros). En este trabajo se adopta la técnica de tratamiento con $\mathrm{Ca}(\mathrm{OH})_{2}$ de acuerdo a las investigaciones desarrolladas por Purwadi et al. (2004) entre otros. Esta técnica consiste en agregar $\mathrm{Ca}(\mathrm{OH})_{2}$ al hidolizado para aumentar el pH de la solución a un valor entre 10-12 manteniendo esta condición por un período de tiempo que se puede extender desde 15 min hasta varios días. Finalmente, se ajusta el $\mathrm{pH}$ a un valor que puede oscilar entre 5 y 7 . Este método presenta como principal desventaja que no sólo se descomponen los productos inhibidores de la fermentación, sino que también se descomponen los azúcares. Por otro lado, cuando se usa $\mathrm{H}_{2} \mathrm{SO}_{4}$ para neutralizar el $\mathrm{pH}$ de la solución ya tratada, precipita $\mathrm{CaSO}_{4}$. 
De acuerdo a nuestro conocimiento, Purwadi et al. (2004) es el único grupo que postula un modelo cinético para la detoxificación. En este aporte se extiende este modelo al incluir la influencia de las variables de proceso tales como el $\mathrm{pH}$ y la temperatura.

La Figura 2 detalla el mecanismo de reacción propuesto, donde cada compuesto A (Furfural, HMF y Azúcares), reacciona con el catión $\mathrm{Ca}^{2+}$ representado por $\mathrm{Z}$, formando los complejos $\{\mathrm{ZA}\}$ que luego se convierten a productos $\mathrm{P}$ ó a las especies originales recuperando parcialmente el $\mathrm{Ca}(\mathrm{OH})_{2}$ utilizado.

Figura 2 - Reacciones químicas de la detoxificación por agregado de $\mathrm{Ca}(\mathrm{OH})_{2}$

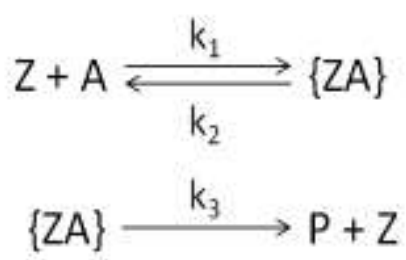

Según indica la técnica explicada arriba, debe limitarse la cantidad de catión $\mathrm{Ca}^{2+}$ presente en la solución de acuerdo al valor de $\mathrm{pH}$ deseado. Finalmente, los balances de masa del proceso se muestran en las Ecuaciones 8-11. El modelo completo de esta etapa se puede encontrar en Moreno, Andersen y Díaz (2013).

$$
\begin{array}{cc}
\frac{d C_{j}}{d t}=-k_{1 j} C_{j} C_{Z}+k_{2 j} C_{Z j} \quad \forall j & \\
\frac{d C_{Z j}}{d t}=-\left(k_{2 j}+k_{3 j}\right) C_{Z j}+k_{1 j} C_{j} C_{Z} \quad \forall j & \quad \forall j \\
\frac{d C_{P j}}{d t}=k_{3 j} C_{Z j} \quad \forall j & \\
\frac{d C_{Z}}{d t}=\sum_{j}\left(k_{2 j}+k_{3 j}\right) C_{Z j}-k_{1 j} C_{j} C_{Z} &
\end{array}
$$

donde $C_{\mathrm{j}}$ representa la concentración del reactante $j\left(j=\mathrm{F}, \mathrm{HMF}\right.$, Azúcares), $C_{Z}$ es la concentración del ión $\mathrm{Ca}^{2+}, C_{Z j}$ representa la concentración del complejo iónico formado en la primer reacción por consumo de cada componente $j$ y los iones de $\mathrm{Ca}^{2+}, C_{P j}$ representa la concentración del producto de la degradación de la sustancia $j$.

El problema DAE en esta etapa consta de 10 ecuaciones diferenciales y 10 ecuaciones algebraicas. El vector de variables diferenciales $\mathbf{z}$ está compuesto por las concentraciones $C_{j}$, $C_{Z}, C_{P}$ y $C_{P j}$. El vector de parámetros $\mathbf{p}$ a estimar tiene en cuenta factores de frecuencia, energías de activación y algunos parámetros relacionados con el cálculo de la cantidad inicial necesaria de $\mathrm{Ca}(\mathrm{OH})_{2}$. 
Los datos experimentales utilizados provienen de Purwadi et al. (2004). En ese trabajo se prolonga la reacción de detoxificación por $90 \mathrm{~min}$, tomando muestras cada $10 \mathrm{~min}$ de los compuestos principales: F, HMF y Azúcares. Este procedimiento se realizó para 4 valores distintos de $\mathrm{pH}\left(9,10,11\right.$ y 12) y para 3 temperaturas diferentes $\left(30^{\circ} \mathrm{C}, 45^{\circ} \mathrm{C}\right.$ y $\left.60^{\circ} \mathrm{C}\right)$, totalizando una cantidad de 12 experiencias.

Finalmente, el modelo DAE cuenta con 120 ecuaciones diferenciales, 9 puntos experimentales para cada ecuación diferencial más la condición inicial y 9 parámetros a estimar. El modelo NLP resultante tiene 11.233 ecuaciones y 11.242 variables.

Los valores de desviación media calculados con los datos experimentales y los valores de concentración óptima, se muestran en la Tabla 3.

Tabla 3 - Valores de desviación media para compuestos que participan de la detoxificación

\begin{tabular}{lccc}
\hline & Azúcares & HMF & Furfural \\
\hline $\begin{array}{l}\text { Desviación } \\
\text { Media }\end{array}$ & $3.1 \%$ & $-0.7 \%$ & $0.3 \%$ \\
\hline
\end{tabular}

Mientras que los valores óptimos de los parámetros se pueden encontrar en la Tabla 4.

Tabla 4 - Valores óptimos de los parámetros más influyentes en el modelo de detoxificación.

\begin{tabular}{cccccc}
\hline Parámetro & Valor óptimo & Parámetro & Valor óptimo & Parámetro & Valor óptimo \\
\hline$E_{1, S}$ & 22,414 & $E_{1, F}$ & 16,532 & $E_{Z}$ & 14,622 \\
$E_{2, S}$ & 66,822 & $E_{3, F}$ & 62,501 & $C_{1}$ & 0,460 \\
$E_{3, S}$ & 63,079 & $E_{1, H M F}$ & 33,510 & $C_{2}$ & 1,000 \\
\hline
\end{tabular}

\section{FERMENTACIÓN SIMULTÁNEA DE HEXOSAS Y PENTOSAS}

Los azúcares de cinco carbonos presentes en el hidrolizado, junto a la glucosa obtenida en la hidrólisis enzimática, son enviados a una etapa de fermentación simultánea denominada co-fermentación. En esta operación, se emplean cepas recombinantes de Zymomonas mobilis capaz de metabolizar ambos azúcares.

Por otro lado, estudios previos demuestran que los compuestos inhibidores de la fermentación como el furfural, pueden ser convertidos por los organismos fermentativos a compuestos menos inhibitorios, sin embargo esta particularidad prolonga la fase lag o de latencia y por lo tanto disminuye la producción de etanol (RANATUNGA et al., 1997).

Leksawasdi et al. (2001) propusieron un modelo de doble sustrato para la cofermentación donde el crecimiento microbiano en cada azúcar se representa por tasas específicas de crecimiento de Z. mobilis como si cada azúcar fuera la única fuente de carbono del proceso. Debido a esto, también introdujeron factores de peso en el consumo de los 
azúcares, forzando a que la suma de ellos fuera igual a la unidad. Estos autores asumieron cinéticas del tipo Monod para la inhibición por sustrato y por producto.

En este artículo se ha extendido el modelo de Leksawasdi et al. (2001) al incluir el efecto inhibitorio del furfural no sólo en el crecimiento de la bacteria Z. mobilis, sino también en el consumo de los sustratos y la formación del producto. Para esto se incluyen nuevos términos en las ecuaciones planteadas. Por otro lado, se asume una cinética de segundo orden para la metabolización del furfural. En las Ecuaciones 12-15 se muestra el balance de masa para la biomasa y el furfural. Todos los balances de masa del sistema se pueden encontrar en Moreno, Andersen y Díaz (2013).

$$
\begin{gathered}
\frac{d C_{x}}{d t}=\left[\alpha r_{x, G}+(1-\alpha) r_{x, X y}\right] C_{x} \\
r_{x, G}=\mu_{\max , G}\left(\frac{C_{G}}{K_{S, G}+C_{G}}\right)\left(1-\frac{C_{E t}-P_{i, G}}{P_{m, G}-P_{i, G}}\right)\left(\frac{K_{i, G}}{K_{i, G}+C_{G}}\right)\left(1-\frac{C_{F}}{C_{F, c r i t}}\right)^{n_{G}} \\
r_{x, X y}=\mu_{\max , X y}\left(\frac{C_{X y}}{K_{S, X y}+C_{X y}}\right)\left(1-\frac{C_{E t}-P_{i, X y}}{P_{m, X y}-P_{i, X y}}\right)\left(\frac{K_{i, X y}}{K_{i, X y}+C_{X y}}\right)\left(1-\frac{C_{F}}{C_{F, c r i t}}\right)^{n_{X y}} \\
\frac{d C_{F}}{d t}=-k_{f} C_{F} C_{x}
\end{gathered}
$$

donde $\alpha$ es el factor de peso para el consumo de glucosa, $\mathrm{r}_{\mathrm{x}, \mathrm{G}}$ y $\mathrm{r}_{\mathrm{x}, \mathrm{Xy}}$ son las velocidades de producción de biomasa. $\mathrm{C}_{\mathrm{j}}$ es la concentración $[\mathrm{g} / \mathrm{L}]$ del compuesto $j$. Los parámetros $\mu_{\max , \mathrm{G}} \mathrm{y} \mu_{\max , \mathrm{X} y}$ simbolizan las tasas máximas de crecimiento del microorganismo sobre la glucosa y xilosa, respectivamente. Los parámetros $\mathrm{K}_{\mathrm{i}, \mathrm{G}}$ y $\mathrm{K}_{\mathrm{i}, \mathrm{Xy}}$ cuantifican la inhibición por sustrato y los parámetros $\mathrm{P}_{\mathrm{m}, \mathrm{G}}$ y $\mathrm{P}_{\mathrm{m}, \mathrm{Xy}}$ representan la concentración máxima de etanol por encima de la cual la bacteria ya no produce etanol. $\mathrm{P}_{\mathrm{i}, \mathrm{G}}$ y $\mathrm{P}_{\mathrm{i}, \mathrm{Xy}}$ son las concentraciones límite de etanol para las fermentaciones de glucosa y xilosa. Los parámetros relacionados con la presencia del furfural son: $\mathrm{C}_{\mathrm{F}, \text { crit }}$ que denota la concentración crítica a la que la inhibición de crecimiento de Z. mobilis es completa, y $\mathrm{n}_{\mathrm{G}} \mathrm{y} \mathrm{n}_{\mathrm{Xy}}$ que son potencias de inhibición. El valor del parámetro $\mathrm{C}_{\mathrm{F}, \text { crit }}$ ha sido calculado en la literatura (RANATUNGA et al., 1997; GUTIERREZ-PADILLA; KARIM, 2005) y equivale a 2,375 [g/L].

El problema DAE completo para la co-fermentación consta de 5 ecuaciones diferenciales. El vector de variables diferenciales $\mathbf{z}$ está compuesto por $C_{\mathrm{X}}, \mathrm{C}_{\mathrm{Xy}}, \mathrm{C}_{\mathrm{G}}, \mathrm{C}_{\mathrm{Et}} \mathrm{y} \mathrm{C}_{\mathrm{F}}$. El vector de parámetros $\mathbf{p}$ está compuesto por 18 elementos.

Los datos experimentales utilizados en la estimación de parámetros provienen de (GUTIERREZ-PADILLA; KARIM, 2005), donde se realizaron 3 experimentos empleando 
diferentes concentraciones iniciales de furfural en el medio de fermentación. El furfural es agregado 10 horas después de la inoculación, entre las fases lag y exponencial. Se tomaron 6 muestras a lo largo de las 40 horas en las que se extiende cada experimento.

Los valores óptimos de los parámetros más influyentes se muestran en la Tabla 5 y los perfiles para cada compuesto en cada experiencia se pueden observar en la Figura 3.

Tabla 5 - Valores óptimos de los parámetros más influyentes en el modelo de co-fermentación

\begin{tabular}{cccc}
\hline Parámetro & Valor óptimo & Parámetro & Valor óptimo \\
\hline$\alpha$ & 0,600 & $P_{m, G}$ & 6,669 \\
$\mu_{\max , G}$ & 0,010 & $P_{m, X y}$ & 9,469 \\
$\mu_{\max , X y}$ & 0,017 & $P_{i, G}$ & 6,575 \\
$K_{i, G}$ & 1,000 & $P_{i, X y}$ & 9,308 \\
\hline
\end{tabular}

Finalmente, el sistema DAE cuenta con 15 Ecuaciones diferenciales y 8 parámetros. El modelo NLP resultante de la aplicación de la técnica de colocación ortogonal cuenta con 320 ecuaciones y 328 variables.

Figura 3 - Perfiles óptimos de concentración vs datos experimentales para glucosa( $\bullet)$, xilosa( $\square)$, etanol $(\boldsymbol{\Delta})$, biomasa $(0)$ y furfural $(\mathrm{X})$
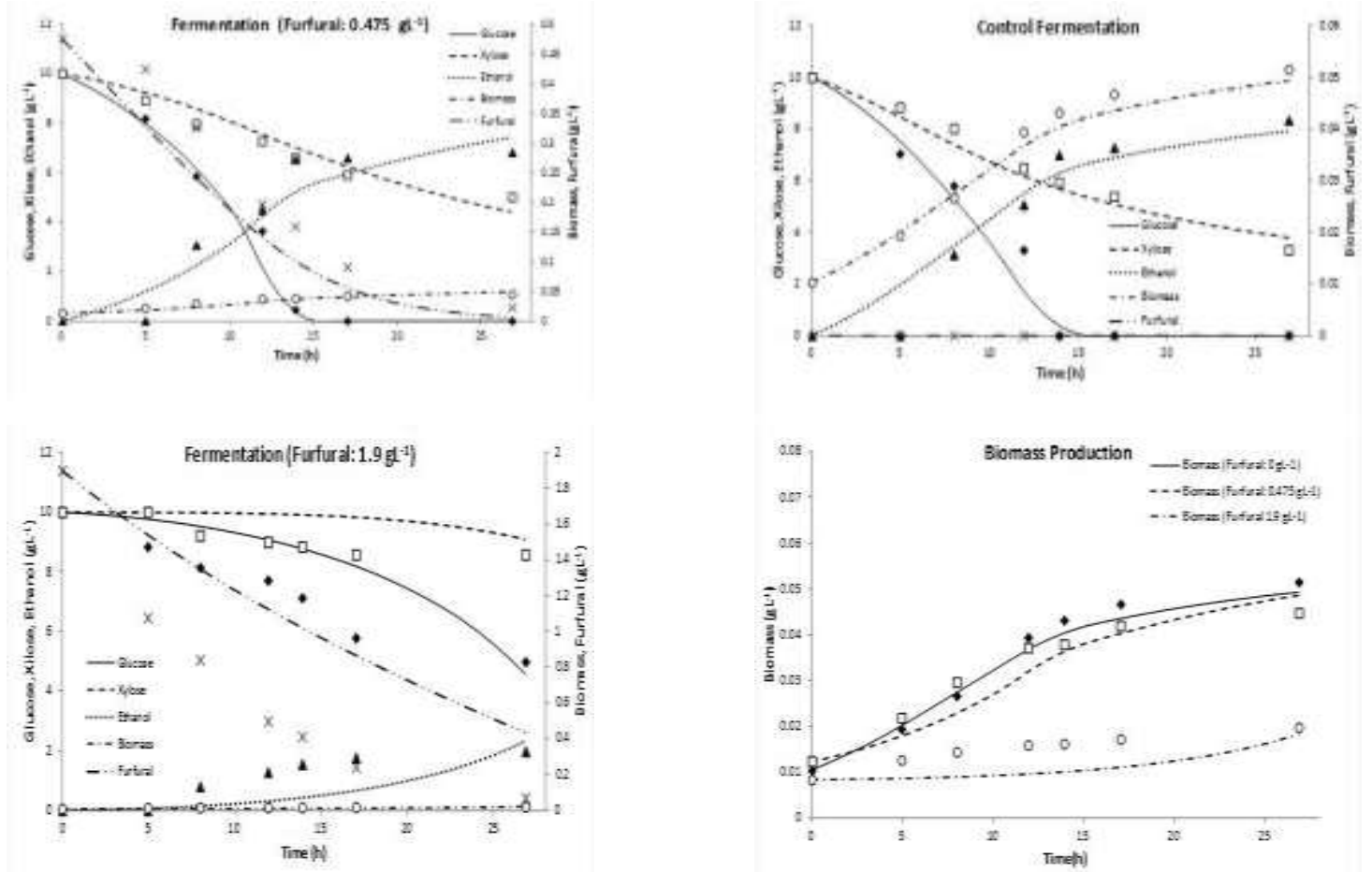

Se puede notar que los mejores ajustes se logran sin la presencia de furfural en el medio. De todas formas, existe una tendencia buena en la representación de los datos para 
concentraciones de furfural distintas de 0 . El último gráfico de la Figura 3 se incluye para visualizar en una escala adecuada los perfiles de crecimiento de biomasa.

\section{CONCLUSIONES}

En el presente estudio se han presentado nuevos modelos y la estimación de parámetros cinéticos para las etapas de hidrólisis ácida diluida, detoxificación y co-fermentación, que son las operaciones principales en la producción de bioetanol por vía bioquímica.

La estimación de parámetros se plantea como un sistema dinámico algebraicodiferencial (DAE) en el que se minimiza una función objetivo de cuadrados mínimos ponderada que se encuentra sujeta a restricciones del proceso.

Este modelo se resuelve mediante un enfoque simultáneo en el que cada sistema DAE es completamente discretizado en el tiempo. Este procedimiento da origen a problemas de optimización NLP que son resueltos con estrategias de punto interior (IP) con programación cuadrática sucesiva (SQP) dentro del programa IPOPT. Para lograr una mayor eficiencia computacional en la resolución del problema, el mismo es desacoplado antes de resolverlo.

Luego, se realiza una vinculación de GAMS con M. Excel para, una vez resuelto el problema, volver a acoplar la información y procesar los resultados automáticamente. De esta forma se logra una representación gráfica que permite dilucidar la bondad del ajuste logrado. Esta representación gráfica corresponde a la mostrada en la Figura 3, que se obtiene instantáneamente después de que GAMS encuentra una solución óptima.

Los parámetros cinéticos se estiman empleando diferentes experiencias reportadas en literatura (PURWADI; NIKLASSON; TAHERZADEH，2004; GUTIERREZ-PADILLA; KARIM, 2005; CASSALES et al., 2011). Los resultados numéricos presentados muestran un ajuste satisfactorio entre los perfiles logrados y los datos experimentales, concluyendo que los modelos propuestos para las etapas de hidrólisis ácida diluida, detoxificación y cofermentación son capaces de describir el comportamiento de los datos experimentales utilizados.

Los modelos propuestos, luego de un escalado apropiado, se utilizarán en futuros trabajos de optimización del proceso de producción de etanol mediante la vía bioquímica. La inclusión de la influencia de furanos tanto en el proceso de co-fermentación como en el de detoxificación, permitirá una optimización estructural determinando la necesidad real de incorporar la etapa de detoxificación dentro del proceso a escala industrial. 


\title{
ETHANOL PRODUCTION FROM LIGNOCELLULOSIC BIOMASS: DYNAMIC PARAMETER ESTIMATION USING A SIMULTANEOUS APPROACH
}

\begin{abstract}
This paper addresses the parameter estimation of kinetic models, describing the unit operations involved in biochemical production of lignocellulosic ethanol. The models are represented by sets of differential-algebraic equations (DAEs), which take into account the main process variables. The parameter estimation problem is solved using a simultaneous approach where state variables are discretized applying orthogonal collocation over finite elements, converting the DAE system into an algebraic one. Thus, the discretization yields a large scale nonlinear programming problem. The estimation of model parameters implies minimizing a weighted least squares objective function subject to algebraic constraints. The kinetic models developed in this paper, can be used in future works about design, simulation, and optimization of ethanol plants. To support this decision, the results obtained within each model have been in good agreement with experimental data from bibliography.
\end{abstract}

Keyword: Parameter estimation. Dynamic optimization. Orthogonal collocation. Lignocellulosic ethanol.

\section{REFERENCIAS}

ANDERSEN, F.; MORENO, M.S.; DÍAZ, M.S. Model-based Optimization of Bioethanol Production from Lignocellulosic Biomass. Proceedings..., AIChE Annual Meeting, 2011.

BIEGLER, L.T.; ZAVALA, V.M. Large-scale nonlinear programming using IPOPT: An integrating framework for enterprise-wide dynamic optimization. Computers \& Chemical Engineering, v. 33, p. 575-582, 2009.

BROOKE, A.; KENDRICK, D.; MEERAUS, A.; RAMAN, R. A User's Guide. 2012, available at the GAMS Development Corporation. Disponible en: 〈http://www.gams.com>.

CASSALES, A.; SOUZA-CRUZ, P.B.; RECH, R.; AYUB, M.A.Z. Optimization of soybean hull acid hydrolysis and its characterization as a potential substrate for bioprocessing. Biomass Bioenergy, v. 35, p. 4675-4683, 2011.

GUTIERREZ-PADILLA, M.G.D.; KARIM, M.N. Influence of furfural on the recombinant Zymomonas mobilis strain CP4 (pZB5) for ethanol production. The Journal of American Science, v. 1, p. 24-27, 2005.

LAVARACK, B.P.; GRIFFIN, G.J.; RODMAN, D. The acid hydrolysis of sugarcane bagasse hemicelluloses to produce xylose, arabinose, glucose and other products. Biomass Bioenergy, v. 23, p. 367-380, 2002.

LEKSAWASDI, N.; JOACHIMSTHAL, E.L.; ROGERS, P.L. Mathematical modeling of ethanol production from glucose/xylose mixtures by recombinant Zymomonas mobilis. Biotechnol. Lett. v. 23, p. 1087-1093, 2001. 
MORENO, M.S.; ANDERSEN, F.; DÍAZ, M.S. Dynamic modeling and parameter estimation for unit operations in lignocellulosic bioethanol production. Industrial \& Engineering Chemistry Research, v. 52, n. 11, p. 4146-4160, 2013.

PURWADI, R.; NIKLASSON, C.; TAHERZADEH, M.J. Kinetic study of detoxification of dilute-acid hydrolyzates by $\mathrm{Ca}(\mathrm{OH}) 2$. Journal Biotechnol, v. 114, p. 187-198, 2004.

RANATUNGA, T.D.; JERVIS, J.; HELM, R.F.; MCMILLAN, J.D.; HATZIS, C. Identification of inhibitory components toxic toward Zymomonas mobilis CP4(pZB5) xylose fermentation. Appl. Biochem. Biotechnol, v. 67, p. 185-198, 1997.

WÄCHTER, A.; BIEGLER, L.T. On the implementation of an interior-point filter line-search algorithm for large-scale nonlinear programming. Math. Program. v. 106, p. 25-57, 2006.

Originais recebidos em: 26/10/2013

Aceito para publicação em: 15/04/2014 